\title{
Coexisting rheumatoid arthritis and ankylosing spondylitis - a case report
}

\author{
Ileana Cosmina Filipescu ${ }^{1,2,3}$, Maria Deac-Badarinza', Laura Muntean ${ }^{1,2,3}$, \\ Simona Rednic ${ }^{1,2,3}$ \\ ${ }^{1}$ Emergency Clinical County Hospital-Rheumatology, Cluj-Napoca, Romania \\ 2"Iuliu Hatieganu" University of Medicine and Pharmacy, Cluj-Napoca, Romania \\ ${ }^{3}$ Centre for Rare Musculoskeletal Autoimmune and Autoinflammatory Diseases, \\ Cluj-Napoca, Romania
}

\begin{abstract}
Rheumatoid arthritis (RA) and ankylosing spondylitis (AS) are chronic progressive inflammatory diseases. Their etiology and symptomatology are different and the coexistence is a rare situation. The new criteria for diagnostic help us to differentiate between those, but a problem may arise when the patient has symptoms typical for both diseases. Early treatment with different disease-modifying antirheumatic drugs and biological agents may delay the progress of these disorders and can cover-up other suggestive symptoms. The purpose of this research is to compare our experience in diagnosis and treatment of concomitant RA and AS with the other researchers' expertise.
\end{abstract}

Keywords: rheumatoid arthritis, ankylosing spondylitis, anti citrullinated peptides antibodies, rheumatoid factor, radiographs

\section{BACKGROUND}

Rheumatoid arthritis (RA) and ankylosing spondylitis (AS) are the most common rheumatic diseases which involve chronic inflammation, progressive joints destructions and disability.

The typical symptoms, serological tests and radiologic changes of AS and RA are totally different, but some clinical manifestations of the two diseases may be similar, including morning stiffness, peripheral arthritis. It is known that genetic predispositions to these diseases are different. The presence of HLADR4 frequently occurs in patients with RA, whereas HLA-B27 predispose to AS (1). Others immunological factors have a high significance. The rheumatoid factor (RF) has been used in the diagnosis of RA since the 1950s and was the first markers used to differentiate between these two disorders (2). In the last years it is useful to determine the level of anti citrullinated peptides antibodies (ACPA), the presence of which increase the sensitivity of the diagnosis of RA (3).

Coexistence of RA and AS in one patient is rare, the first associations was describe in 1976 (4). In the recent years, new diagnostic and classification crite- ria allow more precise differentiation between these two disorders based on the following criteria: ASAS classification criteria for peripheral spondyloarthritis from 2011 and RA classification criteria of the ACR/EULAR (American College of Rheumatology/ European League Against Rheumatism) of 2010 $(5,6)$.

\section{CASE PRESENTATION}

The patient's (non-smoker, female) first symptoms highly suggestive of RA occurred at the age of 29. Two years later had signs of inflammation of symmetric hands joints (metacarpophalangeal MCP and interphalangeal - IFP) and the metatarsophalangeal (MTP) joints of the right foot, as well as a feeling of morning stiffness $>2$ hours. The lab test revealed: mild inflammatory syndrome (erythrocyte sedimentation rate - ESR was $48 \mathrm{~mm} / \mathrm{h}$ and C-reactive protein - CRP was $2.4 \mathrm{mg} / \mathrm{dl}$ ), high values of anti-CCP antibodies ( $64 \mathrm{U} / \mathrm{ml}$; normal $<0.5 \mathrm{U} / \mathrm{ml}$ ) and negative RF. Hands X-ray shows extensive loss of cartilage, small erosion at the left carp, and deformities, compatible with stage III (Fig. 1). 


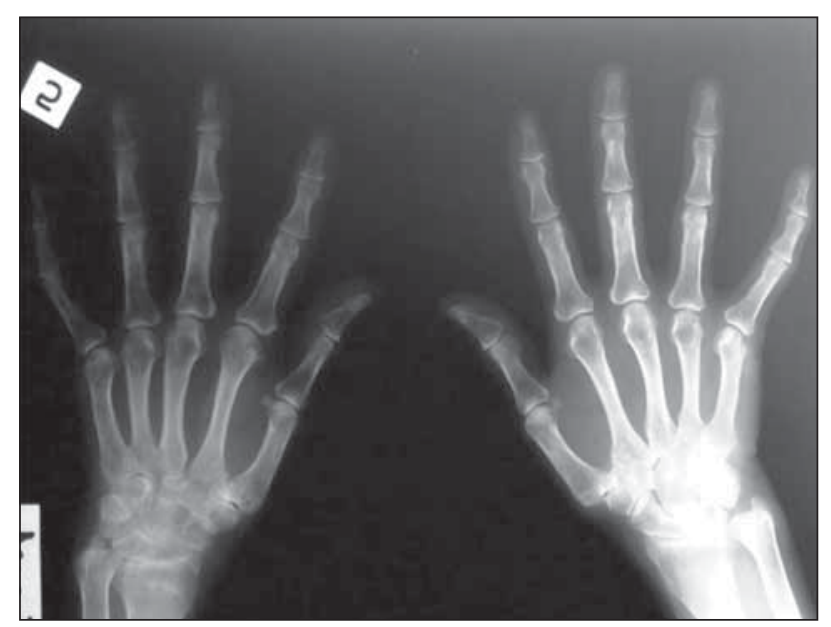

FIGURE 1. Hand X-ray

According to evaluation the symptoms and signs as well as the lab and radiologic examination, the diagnosis was seronegative RA. The treatment was started with different disease-modifying antirheumatic drugs (DMARDs) and biological agents with improvement of the clinical aspects and biological parameters (except CRP, whose value has remained constantly high) (Fig. 2).

After about 12 years the patient reported moderate cervical and back pain, and excessive limitations of the movement of the entire spine. Back pain was of inflammatory nature, exacerbated on the last five months. Morning stiffness persisted for over 1 hour, but without peripheral joints involvement. The phys-

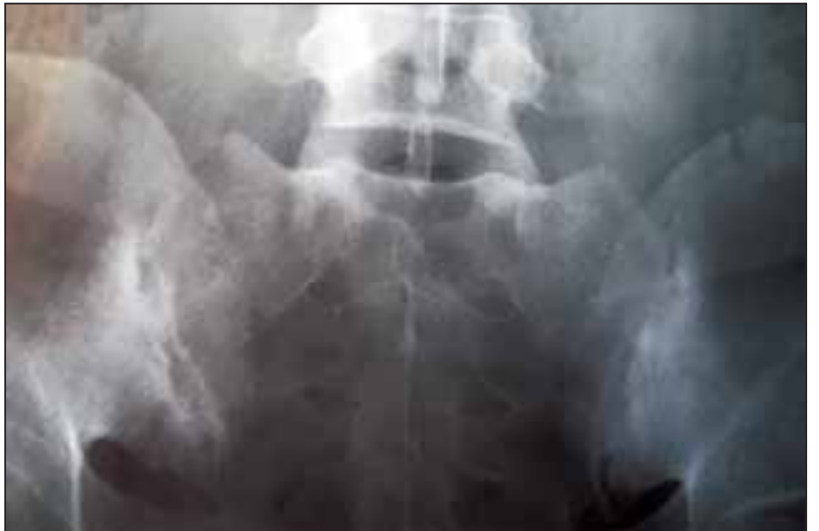

FIGURE 3A. Sacroiliitis on the conventional radiographs

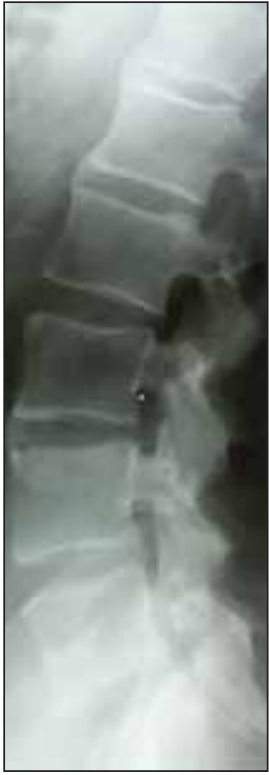

FIGURE 3B. The lumbar spine with syndesmophytes on the radiographs

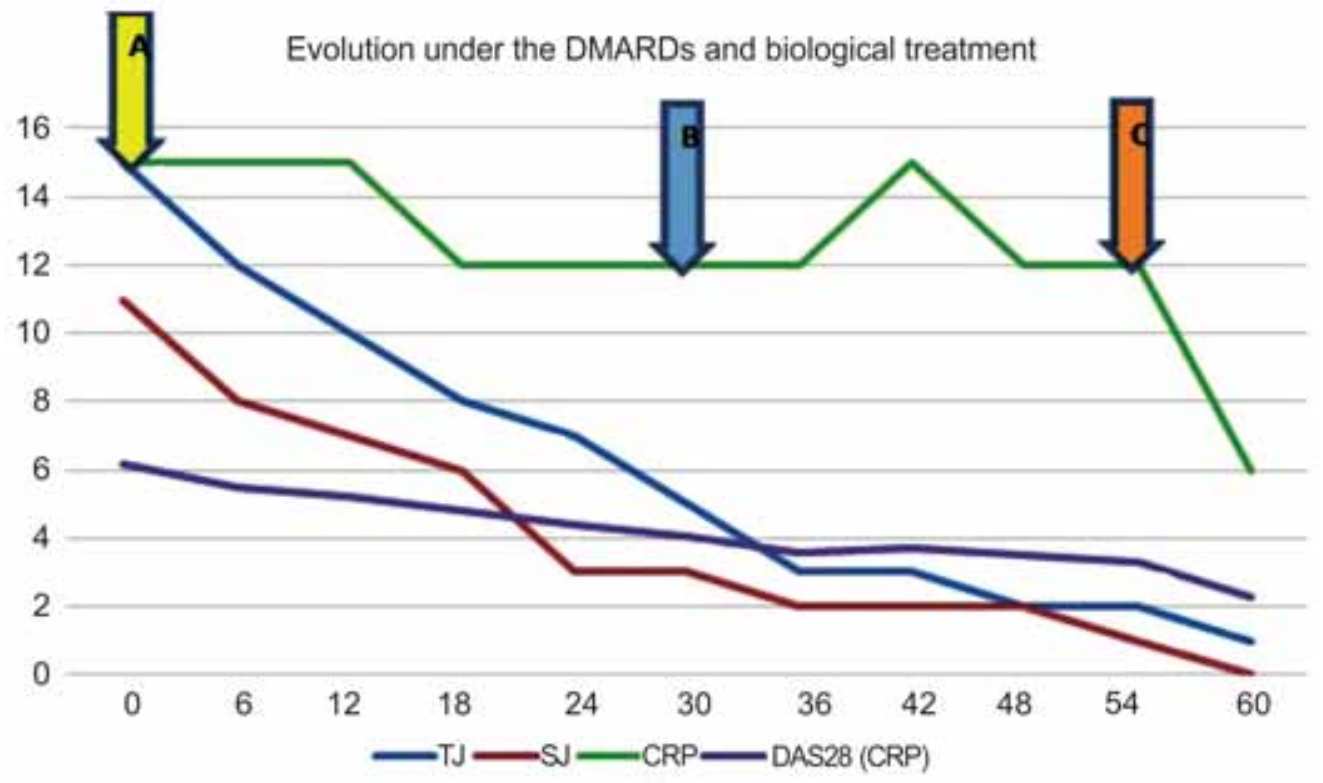

FIGURE 2. Evolution of the case under the remissive treatment

Legend: TJ - tender joint, SJ - swollen joint, CRP - C reactive Protein, DAS28 - The Disease Activity Score 28, A - First biological agent (Remicade), B - The second biological agent (Etanercept), C - The third biological agent (Cimzia) 
ical examination revealed pain and arthritis only on the left MCF II joint, ulnar deviation of the fingers, atrophy of the interosseous muscles of the both hands, decreased mobility of left wrist joints. Furthermore, limited spinal mobility was observed, mainly in the cervical and chest section: turn 20 degrees right, 25 degrees left, the occiput-to-wall distance: $8 \mathrm{~cm}$, chest respiratory expansion: $1.0 \mathrm{~cm}$, kyphosis of the thoracic spine, paravertebral muscle contracture, Schober's test: $2 \mathrm{~cm}$, flattening of the lumbar lordosis, fingertip-to-floor distance: $25 \mathrm{~cm}$, intermalleolar distance: $78 \mathrm{~cm}$.

The value of CRP was elevated $(1.2 \mathrm{mg} / \mathrm{dl}$; normal $<0.5$ ) with a normal ESR.

Radiological examination of the spine and SI joints reveal the presence of syndesmophytes and sacroiliitis stage III (Fig. 3). Presence of HLA-B27 was confirmed. Screening test for osteoporosis was negative ( $\mathrm{T}$ - Score on DXA was - 0.6). The others examination to exclude hidden inflammations were negative (screening for infections and neoplasia, fat biopsy for amyloidosis, etc.).

\section{DISCUSSION}

In the systematic literature female sex is associated with a greater likelihood of developing RA, and male sex is associated with a greater likelihood of developing AS (4). RA is usually diagnosed at the age of 40-50, whereas symptoms characteristic of AS start appearing before the age of 30 (4-6).

Epidemiological data, as well as higher incidence of both RA and AS in certain families and identical twins, support the significance of genetic factors. Approximately $60 \%$ of the risk of RA may be attributed to genetic factors, especially HLA-DR1 and HLA-DR4 (7). These relationship was confirmed for AS and HLA-B27 (4). No relationship was found between HLA-B27 and RA. Toussirot and colleague found that $6.6 \%$ of patients with RA may have positive HLA-B27 which is comparable to the general population, wherein the expression of this antigen is $5-8 \%(8)$. They show that the presence of HLA-B27 in patients with RA does not increase the incidence of enthesitis or sacroiliac joint inflammation (8-10). Only 5\% of the people with positive HLA-B27 can develop AS but this antigen is detected in up to $90-95 \%$ of Caucasians suffering from AS (1). This explains the increased role of environmental factors in the occurrence of the disease in genetically predisposed individuals.
In our subject the presence of HLA B27 was confirmed, but it was not possible to identify the presence of HLA DR4.

Some studies have suggested that there is no erosive polyarthritis in RF positive AS patients, and sacroiliac joint involvement is not commonly seen in RA patients who are HLA-B27 positive $(8,9)$.

In both disorders many pro-inflammatory cytokines are overproduced, such as tumor necrosis factor (TNF). This explains the efficacy of the treatment with inhibitors of this cytokine in RA and SA (11). There are some cases of RA coexisting with AS treated with tocilizumab $(11,12)$. In 2014, Dundar et al. described a case of female patient diagnosed with coexisting RA and AS treated with rituximab with good effect (13).

Our patient had a good response to the anti-TNF $\alpha$ inhibitors (Remicade, Etanercept, Cimzial) and was in remission (based on DAS28 - ESR). The persistent elevated CRP values, in the absence of other identified causes, the occurrence of static vertebral disorders with marked limitation of mobility raised the suspicion of coexistence in this case between RA and SA.

In the typical case of RA, the most characteristic symptom is symmetrical inflammation of numerous small joints, however patients with AS may also present peripheral arthritis (in about one third of cases), usually asymmetric and typically involving a single large joint, although small joints may also be affected $(4,5)$. Typical radiological aspects in patients with RA revealed destruction of articular cartilage and bone in the form of erosions and periarticular osteoporosis, and later joint distortion and bone fusions. These changes are located within the small joints and appear especially in the early stages of an active RA. Characteristic features of AS are calcifications in tissues near the spine, appearance of the syndesmophytes and bone fusions. These abnormalities concern the spine and sacroiliac joints. The typical radiological image is used to confirm the diagnosis of both diseases $(3,4)$. Erosions are rarely observed in patients with AS $(7,11)$. In our subject the erosions appeared in the first 2 years. In RA, sacroiliac joint inflammation is rare (up to $20 \%$ of the patients) (4). The changes are located in distal part of the sacroiliac joint and the degree of damage is lower (4). The typical inflammatory changes in the spine in patients with RA are most often observed in the upper cervical spine, affected the atlanto-occipital and atlanto-axial joint, the typical syndesmophytes was not observed (13). 
The typical serological markers characteristic of RA include the RF and ACPA. RF occurs in $70-85 \%$ of cases of RA (2-4). It is characterized by low specificity. The frequency of the RF in the general population increases with age and may be detected in many other diseases $(2,3)$. In principle it is absent in seronegative spondylarthritis ( $\mathrm{SpA})$. Tuossirot et al. found that the RF was positive in $8.3 \%$ of patients with AS and in $9.8 \%$ of the control group (not statistically significant) (8). A much more specific marker of RA is antibodies against citrullinated peptides, observed in $80-92 \%$ of patients with RA (13-15).

A review of the literature in 1995 described 44 similar cases (4). According to these authors, a typical patient with coexisting RA and AS is a man who developed axial SpA at the age of 20-30, and who developed symptoms of peripheral arthritis after a few years. Only 10 patients developed the symptoms of RA at the beginning. The probability of developing both diseases was estimated at 1:100,000 $(8,16)$.

However, it is rare for two types of disease to occur over a long period of time (12 years) as we reported in this work. Barczyńska et al in 2015 presents also a case with RA-SA diagnosed over 6 years (4).

In 2011, Guo et al. described a 30-year-old female patient diagnosed with coexisting RA-AS and referred to 20 other such patients from China (17).

The authors of these articles assume that pregnancy might be one of the environmental factors that induced both diseases in individuals with a positive HLA-B27 and HLADR4 (17).
The presence of other symptoms characteristic of SpA may increase the sensitivity to diagnosed SA. In 2013, Can et al. determined the incidence of IBP and other symptoms in a group of 167 RA (18). The criteria for IBP were met in $16.8 \%$ of patients and enthesitis was reported in $41.9 \%$ of patients (18). X-ray showed features of bilateral sacroiliitis grade 3 in 4 patients (2.4\%). All patients with coexistence of RA and AS had positive RF and anti-CCP (18).

Ying-ying and colleague suggest that even if the symptoms of RA are highlighted, especially in the young patient with RA and extra-articular symptoms compatible with AS and hip joints disorders (e.g., iridocyclitis, sinus bradycardia), it is better to ask the patient about the low-back pain. In this case the HLA-B27 test and radiologic examination of the sacroiliac joint should be performed to discover if AS and RA coexist (17). To exclude RA association, they also recommended testing for the HLA-DR4 gene and ACPA to be performed for the patient with AS and symptoms at the peripheral joints (17).

\section{CONCLUSIONS}

Arthritis may be present in many rheumatic disorders, one diagnosis does not necessarily exclude another, coexistence of two or even more inflammatory joint diseases in the same patient may result in modification of the clinical image and an inadequate response to the treatment. Sometimes, after many years, we need to verify a previous diagnosis.

Conflict of interest: none declared Financial support: none declared

\section{REFERENCES}

1. Maksymowych W.P., Brown M.A. Genetics of ankylosing spondylitis and rheumatoid arthritis: where are we at currently, and how do they compare? Clin Exp Rheumatol 2009; 27(4 Suppl 55): S20-S25

2. Song Y.W., Kang E.H. Autoantibodies in rheumatoid arthritis: rheumatoid factors and anticitrullinated protein antibodies. QJM. 2010 Mar; 103(3): 139-146.

3. Toes R.E., van der Woude D. ACPA (anti-citrullinated protein antibodies) and rheumatoid arthritis. Acta Reumatol Port. 2011 JulSep; 36(3):205-7.

4. Barczyńska T.A., Węgierska M., Żuchowski P. et al. Coexistence of rheumatoid arthritis and ankylosing spondylitis. Reumatologia. 2015; 53(5): 279-285.

5. Rudwaleit M., van der Heijde D., Landewé R. et al. The Assessment of SpondyloArthritis International Society classification criteria for peripheral spondyloarthritis and for spondyloarthritis in general. Ann Rheum Dis. 2011; 70(1):25-31.

6. Aletaha D., Neogi T., Silman A.J. et al. 2010 Rheumatoid arthritis classifcation criteria: an American College of Rheumatology/ European League Against Rheumatism collaborative initiative. Arthritis Rheum 2010; 62(9):2569-81
7. van der Horst-Bruinsma I.E., Lems W.F., Dijkmans B.A. et al. A systematic comparison of rheumatoid arthritis and ankylosing spondylitis. Clin Exp Rheumatol 2009; 27 (4 Suppl 55): S43-S49.

8. Toussirot E., Acquaviva P.C. Coexisting rheumatoid arthritis and ankylosing spondylitis discussion of 3 cases with review of the literature. Clin Rheumatol 1995; 14:554-560.

9. Mera-Varela A., Ferreiro-Iglesias A., Perez-Pampin E. et al. Ultrasonographic assessment of enthesitis in HLA-B27 positive patients with rheumatoid arthritis, a matched case-only study. PLoS One 2013; 8: e58616.

10. Can G., Solmaz D., Binicier O. et al. High frequency of inflammatory back pain and other features of spondyloarthritis in patients with rheumatoid arthritis. Rheumatol Int 2013; 33: 12891293.

11. Appel H., Loddenkemper C., Miossec P. et al. Rheumatoid arthritis and ankylosing spondylitis - pathology of acute inflammation. Clin Exp Rheumatol 2009; 27 (4 Suppl 55): 15-19.

12. Baksay B., Dér A., Szekanecz Z. et al. Coexistence of ankylosing spondylitis and rheumatoid arthritis in a female patient. Clin Rheumatol 2011; 30: 1119-1122. 
13. Dundar U., Cevik H., Demirdal U.S. et al. Use of rituximab to treat a patient with coexistence of rheumatoid arthritis and ankylosing spondylitis: 18 months follow-up. Int J Rheum Dis 2014; doi: 10.1111/1756-185X.12303.

14. Seegobin S.D., Ma HYM, Dahanayake C. et al. ACPA-positive and ACPA-negative rheumatoid arthritis differ in their requirements for combination DMARDs and corticosteroids: secondary analysis of a randomized controlled trial. Arthritis Research \& Therapy 201416:R13.

15. van Heemst J., Trouw L.A., Nogueira L. et al. An investigation of the added value of an ACPA multiplex assay in an early rheumatoid arthritis setting. Arthritis Res Ther. 2015 Oct 5; 17:276
16. Joshua V., Schobers L., Titcombe P.J. et al. Antibody responses to de novo identified citrullinated fibrinogen peptides in rheumatoid arthritis and visualization of the corresponding B cells. Arthritis Res Ther. 2016 Dec 1; 18(1):284.

17. Ying-ying G., Li-li Y., Hua-dong C. et al. Coexisting ankylosing spondylitis and rheumatoid arthritis: A case report with literature review. Chin Med J 2011; 124(20):3430-3432.

18. Can G., Solmaz D., Binicier O. et al. High frequency of inflammatory back pain and other features of spondyloarthritis in patients with rheumatoid arthritis. Rheumatol Int 2013; 33: 12891293 